Artigo original

Hegemonia - Revista Eletrônica de Relações Internacionais do Centro Universitário Unieuro

ISSN: $1809-1261$

UNIEURO, Brasília, número 16, 2015, pp. 46-67.

Recebido em: 20/4/2015

Avaliado em:12/5/2015

Aprovado em: 18/6/2015

\title{
CONSUMO FREQUENTE DE CARNE VERMELHA E PROCESSADA E SUA ASSOCIAÇÃO COM O DESENVOLVIMENTO DE DOENÇAS CRÔNICAS NÃO TRANSMISSÍVEIS: UMA ANÁLISE A PARTIR DA SAÚDE COLETIVA
}

\author{
Luana Galeno dos Santos, ${ }^{1}$ \\ Aldira Guimarães Duarte Dominguez, ${ }^{2}$ \\ e Vanessa Resende Nogueira Cruvinel ${ }^{3}$
}

RESUMO: As Doenças Crônicas Não Transmissíveis (DCNT) são as principais causas de morbimortalidade no mundo, e atualmente são consideradas um sério problema de saúde pública. Dentre as principais DCNT destacam-se as cardiovasculares, câncer e diabetes. Estas doenças caracterizam-se por acompanharem seus portadores por tempo indeterminado podendo se desenvolver a partir de fatores endógenos e exógenos. Estes problemas são associados com uma dieta inadequada, especialmente com o consumo de carne Saúde Coletiva.

Sanitarista formada pela Universidade de Brasília, Faculdade de Ceilândia, Curso de

Professora Doutora Adjunta III da Universidade de Brasília, Faculdade de Ceilândia, Curso de Saúde Coletiva.

Professora Doutora Adjunta II da Universidade de Brasília, Faculdade de Ceilândia, Curso de Saúde Coletiva. 
Artigo original

Hegemonia - Revista Eletrônica de Relações Internacionais do Centro Universitário Unieuro

ISSN: 1809-1261

UNIEURO, Brasília, número 16, 2015, pp. 46-67.

vermelha e processada. Neste sentido, esse estudo tem como objetivo identificar na literatura a associação entre o consumo frequente de carne vermelha e processada e o desenvolvimento de DCNT. Para tanto, realizou-se uma pesquisa exploratória a partir de uma busca bibliográfica nas bases de dados (SciELO, PubMed e LILACS). Após aplicados os critérios de inclusão e exclusão estabelecidos para a busca bibliográfica, restaram ao total onze artigos no idioma inglês que abordavam o objeto de estudo. Os resultados demonstraram que o consumo frequente de carne vermelha e processada pode desencadear diabetes tipo 2, doenças cardiovasculares e cânceres, principalmente o colorretal e de esôfago. Encontrou-se também como resultado que as dietas que contêm grande quantidade de legumes, frutas e hortaliças podem tanto ser um fator de proteção contra essas doenças, quanto um determinante para o controle. Percebe-se que há uma necessidade de que as pessoas reduzam o consumo de carne vermelha e processada na alimentação em prol da sua saúde. Ações pensadas na perspectiva da Saúde Coletiva são de fundamental importância, para diminuir esses tipos de problemas correlacionados com o consumo de carne vermelha e processada.

Palavras-chave: Diabetes tipo 2; Doenças cardiovasculares; Câncer; Consumo de carne.

ABSTRACT: The Chronic Noncommunicable Diseases (NCDs) are the principal causes of morbidity and mortality in the world. Actually these diseases are considered a serious public problem health. The main NCDs are cardiovascular, cancer and diabetes. These diseases follow the victims during an undetermined time, developping their symptom through endogens and exogens factors. These problems are associate with a poor diet and especially with processed meat consumptions. In this way, will be performed studies in order to identify the association between regular consumption of meat red and processed meat and the development of NCDs. Therefore, we carried out an exploratory study from a literature search in databases (SciELO, PubMed e LILACS). After applying inclusion and exclusion criteria established for the literature search, in total remains eleven articles in the english language that addressed the object of study. The results demonstrated that regular consumption of red meat and processed may initiate type 2 diabetes, cardiovascular diseases and cancers, 
Artigo original

Hegemonia - Revista Eletrônica de Relações Internacionais do Centro Universitário Unieuro

ISSN: $1809-1261$

UNIEURO, Brasília, número 16, 2015, pp. 46-67.

particularly colorectal and esophagus. It was also found as a result that diets that contain lots of vegetables, fruits and vegetables can either be a protective factor against these diseases, as a key to control the problems. Based in these recent data, it is necessary that people seek to reduce the meat consumptions in order to increase their life quality. In this way, collective actions in Coletive Health are very important to decrease these kind of problems correlated with red and processed meat consumptions.

Keywords: Type 2 diabetes; Cardiovascular diseases; cancer; Meat consumption.

INTRODUÇÃO

A prática alimentar de uma sociedade permeia questões econômicas, sociais e culturais, e de acordo com Mota (2005, p. 9), "seis entre as dez principais causas de morte no mundo estão ligadas à alimentação, sendo elas: doenças cardíacas, câncer, derrame, diabetes, problemas crônicos, do fígado e arteriosclerose", e uma das características dessas doenças é que são Doenças Crônicas Não Transmissíveis (DCNT).

As DCNT são caracterizadas por acompanharem seus portadores por tempo indeterminado e demandam constantemente de suporte de uma equipe de saúde interdisciplinar, assim como de medicamentos, exames específicos, ademais de estratégias e ações para a sua prevenção e autocuidado para com sua saúde. De acordo com o Ministério da Saúde (2014) "as doenças crônicas não transmissíveis são doenças multifatoriais que se desenvolvem no decorrer da vida e são de longa duração. Atualmente, elas são consideradas um sério problema de saúde pública".

A Organização Mundial da Saúde (OMS, 2013), diz que 63\% das mortes no mundo são causadas por DCNT, o que equivale a 36 milhões de mortes por ano, principalmente em países em desenvolvimento.

O desenvolvimento das DCNT como câncer, diabetes tipo 2 e doenças cardiovasculares estão associados a fatores de risco, como a inatividade física, alimentação inadequada, consumo nocivo de álcool e tabagismo (BRASIL, 2011). A redução destes fatores de risco diminui de forma significativa o risco do aparecimento dessas doenças, legitimando políticas e programas para 0 
Artigo original

Hegemonia - Revista Eletrônica de Relações Internacionais do Centro Universitário Unieuro

ISSN: $1809-1261$

UNIEURO, Brasília, número 16, 2015, pp. 46-67.

enfrentamento destes hábitos inadequados. Nessa lógica, o presente estudo tem por base uma busca por artigos científicos a fim de saber a associação entre 0 consumo frequente da carne vermelha e processada e 0 desenvolvimento dessas DCNT.

Neste estudo entende-se por consumo frequente, a ingestão de quatro a seis porções de carne vermelha e processada por semana. (ASSUNÇÃO et al, 2012). Por carne vermelha, define-se como as bovinas, e carnes processadas como sendo: bacon, salsicha, nuggets, linguiça, presunto dentre outros (CROSS et al, 2007).

Apesar de hoje já existir, principalmente no idioma inglês, literatura que aborda abertamente o consumo excessivo de carne vermelha e processada com o risco de adoecimento, a motivação dessa pesquisa é pautada na percepção da falta de divulgação dessas informações que, muitas vezes não chegam ao conhecimento dos indivíduos e acabam por não refletir sobre seus hábitos alimentares.

Assim, este estudo tem como propósito ser mais uma opção de esclarecimento e provável sensibilização para o autocuidado da saúde dos indivíduos e consequentemente menos procura por assistência nos serviços de saúde.

A percepção do consumo de carne vermelha e processada como o risco de adoecimento é mais notório nas pessoas sensibilizadas para o tema, como: vegetarianos, veganos, alguns nutricionistas, alguns cardiologistas, dentre outros. A ideia é ampliar o debate sobre o tema, fazendo com que as informações sobre os riscos cheguem até a população possibilitando uma reflexão, e visando uma redução desse consumo pelo menos em alguns dias da semana. Espera-se também que com este estudo novas pesquisas sejam fomentadas, principalmente no campo da Saúde Coletiva, para que decisões mais assertivas sejam tomadas especialmente no que se refere à formulação e orientação das políticas públicas.

\section{OBJETIVOS}

O objetivo geral foi identificar na literatura a associação entre o consumo frequente de carne vermelha e processada com o aparecimento de DCNT. E os 
Artigo original

Hegemonia - Revista Eletrônica de Relações Internacionais do Centro Universitário Unieuro

ISSN: $1809-1261$

UNIEURO, Brasília, número 16, 2015, pp. 46-67.

específicos foram realizar busca bibliográfica nas bases de dados SciELO, PubMed e LILACS, a procura de artigos científicos que associem o tema de DCNT relacionadas com consumo de carne vermelha e processada; e contribuir à reflexão sobre o consumo frequente de carne vermelha e processada e seus malefícios.

Procedimentos Metodológicos

Para a realização desse trabalho foi realizado uma pesquisa exploratória, por meio de uma revisão bibliográfica. Gil (2008, p. 27), diz que "as pesquisas exploratórias têm como principal finalidade desenvolver, esclarecer e modificar conceitos e ideias, tendo em vista a formulação de problemas mais precisos ou hipóteses pesquisáveis para estudos posteriores".

Conforme Gil (2008, p. 50), "a pesquisa bibliográfica é desenvolvida a partir de material já elaborado, constituído principalmente de livros e artigos científicos".

Ainda de acordo com o autor:

Pesquisas exploratórias são desenvolvidas com o objetivo de proporcionar visão geral, de tipo aproximativo, acerca de determinado fato. Este tipo de pesquisa é realizado especialmente quando o tema escolhido é pouco explorado e torna-se difícil sobre ele formular hipóteses precisas e operacionalizáveis (GIL, 2008, p. 27).

Procedimentos para coleta de dados

Foram utilizadas as bases de dados SciELO (Scientific Electronic Library Online), PubMed (U. S. National Library of Medicine) e LILACS (Literatura Latino-Americana e do Caribe em Ciências da Saúde). As bases de dados citadas são de acesso público e gratuito, sendo os dados para coletados no mês de março de 2015.

Os termos empregados para fazer a busca foram: carne vermelha and câncer, carne vermelha and diabetes tipo 2, carne vermelha and doenças cardiovasculares, carne processada and câncer, carne processada and diabetes tipo 2, carne processada and doenças cardiovasculares. No idioma inglês, os 
Artigo original

Hegemonia - Revista Eletrônica de Relações Internacionais do Centro Universitário Unieuro

ISSN: $1809-1261$

UNIEURO, Brasília, número 16, 2015, pp. 46-67.

termos foram: red meat and cancer, red meat and type 2 diabetes, red meat and cardiovascular diseases, processed meat and cancer, processed meat and type 2 diabetes, processed meat and cardiovascular diseases.

Critérios de inclusão e exclusão

Os critérios de inclusão foram: artigos de fontes primárias de estudo; idioma português ou inglês; e textos na íntegra, de forma online e gratuita. Os critérios de exclusão foram: artigos de revisões de literatura e revisões sistemáticas; artigos com texto incompleto; artigos de outro idioma não sendo o português ou inglês; e artigos que não respondiam ao objeto de estudo.

Não houve limitação de ano, sendo incluídos todos os artigos científicos que respondessem aos objetivos desta pesquisa, tendo em vista que há poucos estudos sobre o tema, fazendo-se necessário, portanto, a abrangência de todos os anos. Os idiomas escolhidos foram o português e o inglês. A coleta de dados foi realizada no mês de março de 2015

Esta pesquisa não passou pelo Comitê de Ética em Pesquisa (resolução n0466/12) para a realização, visto que os dados encontrados estão disponíveis na internet para acesso livre e gratuito. Os dados coletados foram unicamente utilizados para fins científicos.

\section{RESULTADOS E DISCUSSÃO}

Usando os termos citados na metodologia para a busca dos artigos, foram encontrados ao total 144 artigos, sendo o maior número deles encontrados na PubMed com 96 achados, SciELO 26 e LILACS 22. Aplicados os critérios de inclusão e exclusão restaram 11 artigos no idioma inglês, sendo que 2 falam sobre doenças cardiovasculares, 2 falam sobre diabetes mellitus tipo 2, e 7 falam sobre câncer. Esta pesquisa é de caráter exploratório, e espera-se que através desta, outros estudos mais aprofundados e com a inclusão de outras doenças crônicas não transmissíveis sejam realizadas. 


\section{Artigo original}

Hegemonia - Revista Eletrônica de Relações Internacionais do Centro Universitário Unieuro

ISSN: $1809-1261$

UNIEURO, Brasília, número 16, 2015, pp. 46-67.

\section{Diabetes Tipo 2}

A Sociedade Brasileira de Diabetes - SBD define diabetes como sendo a elevação da glicose no sangue, chamada de hiperglicemia, devido à produção deficiente ou a falta do hormônio insulina produzido nas células do pâncreas. Classifica ainda 3 tipos de diabetes: tipo 1, tipo 2 e gestacional (SBD, 2014).

\section{Diabetes Tipo 1:}

É também conhecido como diabetes insulinodependente, diabetes infanto-juvenil e diabetes imunomediado. Neste tipo de diabetes a produção de insulina do pâncreas é insuficiente pois, suas células sofrem o que chamamos de destruição autoimune. [...] 0 diabetes tipo 1 embora ocorra em qualquer idade é mais comum em crianças, adolescentes ou adultos jovens (SBD, 2014)..

Diabetes Tipo 2:

É também chamado de diabetes não insulinodependente ou diabetes do adulto e corresponde a $90 \%$ dos casos de diabetes. Ocorre geralmente em pessoas obesas com mais de 40 anos de idade embora na atualidade se vê com maior frequência em jovens, em virtude de maus hábitos alimentares, sedentarismo e stress da vida urbana [...] Por ser pouco sintomática o diabetes na maioria das vezes permanece por muitos anos sem diagnóstico e sem tratamento o que favorece a ocorrência de suas complicações no coração e no cérebro (SBD, 2014).

Diabetes Gestacional:

Durante a gravidez ocorrem adaptações na produção hormonal materna para permitir o desenvolvimento do bebê. A placenta é uma fonte importante de hormônios que reduzem a ação da insulina, responsável pela captação e utilização da glicose pelo corpo. O pâncreas materno, consequentemente, aumenta a produção de insulina para compensar este quadro de resistência á sua ação (SBD, 2014). 
Artigo original

Hegemonia - Revista Eletrônica de Relações Internacionais do Centro Universitário Unieuro

ISSN: $1809-1261$

UNIEURO, Brasília, número 16, 2015, pp. 46-67.

Os principais sintomas desta doença são: poliúria, polidipsia, polifagia, e fadiga intensa (DULLIUS, 2007).

Para este estudo tomou-se como base a diabetes tipo 2, por esta ser encontrada com mais frequência na população em geral e por ter como um de seus fatores de risco a alimentação inadequada.

Geralmente associa-se 0 aparecimento de diabetes tipo 2, com hereditariedade, obesidade e sedentarismo, esses fatores não estão errados, porém não são os únicos (ORTIZ; ZANETTI, 2001; MEDEIROS et al, 2012). Normalmente, poucas pessoas associam o consumo de carne vermelha e processada ao risco de desenvolvimento de diabetes tipo 2. No entanto, evidências científicas correlacionam este fator de risco à doença.

Os estudos de coorte prospectivo ${ }^{4}$ de Dan et al (2002) e Männistö et al (2010), demonstraram que o consumo de carne processada possivelmente está ligada ao desenvolvimento de diabetes tipo 2 .

Para investigar se havia relação entre o consumo de carne e o risco de diabetes tipo 2, Dan et al (2002), durante os anos de 1986 a 1998, acompanharam profissionais de saúde do sexo masculino por meio de avaliações dietéticas, Índice de Massa Corporal (IMC) ${ }^{5}$, atividade física e consumo de gorduras trans, os participantes estavam inseridos nas seguintes carreiras: veterinários, dentistas, farmacêuticos, podólogos, optometristas e médicos osteopatas.

Para este estudo foram excluídos os homens que relataram ter diabetes, algum tipo de câncer e doenças cardiovasculares para que isso não afetasse na dieta deles. Os integrantes da pesquisa foram acompanhados por 12 anos,

. Nos estudos de coorte, prospectivos, o investigador inicia o trabalho com um grupo de indivíduos aparentemente livres da doença. Este grupo de indivíduos, ou coorte, é dividido em expostos, não-expostos a possíveis fatores de risco, sendo então acompanhados no tempo para determinar a taxa de incidência de determinada doença ou mortalidade entre os grupos. (PITANGA, 2002, p. 52)

Índice de Massa Corporal (IMC), é calculado da seguinte forma: peso/estatura . (MELLO et al, 2004, p. 175). 
Artigo original

Hegemonia - Revista Eletrônica de Relações Internacionais do Centro Universitário Unieuro

ISSN: $1809-1261$

UNIEURO, Brasília, número 16, 2015, pp. 46-67.

sendo que a cada dois anos respondia-se um questionário para identificar casos de diabetes e/ou outras doenças, assim como, peso, casos de tabagismo e prática de atividades físicas. E em anos específicos respondiam um questionário semiquantitativo para atualizar a dieta e indicar quantas vezes consumiram 0 total de cada alimento (DAN et al, 2002).

Os resultados deste estudo mostraram que os integrantes que consumiam mais gordura saturada possuíam também um menor nível de atividade física, IMC elevado, tinham hipercolesterolemia, assim como, ingeriam menos quantidade de fibras e cereais.

Obtiveram também como resultados, que o consumo de carne processada contribuiu para uma ingestão elevada de gordura, e que "homens que consumiram carne processada, pelo menos, cinco vezes por semana tiveram um risco relativo mais elevado para desenvolver diabetes tipo 2 comparado com os homens que consumiram carnes processadas menos de uma vez por mês" (DAN et al, 2002, p. 421, tradução nossa).

Os tipos de carne processadas que estavam significativamente ligada ao risco de diabetes tipo 2 eram: Bacon, salsicha, nuggets, outras carnes processadas e hambúrgueres.

Por fim, o estudo conclui que o consumo frequente de carne processada, foi associado com um aumento do risco de diabetes tipo 2 (DAN et al, 2002.). Relaciona-se o consumo de carne processada como um desencadeador de DCNT, devido à alta quantidade de colesterol - LDL, sal, e por ser um alimento de baixo valor nutricional (ASSUNÇÃO et al, 2012).

Uma ingestão elevada de colesterol LDL é associada ao risco de desenvolvimento de doenças, ao passo que, dentro do organismo atua nas paredes internas dos vasos sanguíneos, favorecendo o depósito de gorduras nas artérias, causando o estreitamento e prejudicando o fluxo sanguíneo. Há ainda a recomendação para que a ingestão de carnes vermelhas e processadas seja de forma moderada, dando preferência aos alimentos com menores teores de açúcar (BRASIL, 2006b).

O estudo de Dan et al (2002), não excluiu os fumantes e nem teve como pré requisito que os participantes fossem tabagista, constatando apenas que, os que ingeriam mais gordura saturada eram os mais propensos a fumar cigarros.

O estudo de Männistö et al (2010), teve por objetivo identificar se o consumo de carne vermelha e processada teria relação com desenvolvimento 
Artigo original

Hegemonia - Revista Eletrônica de Relações Internacionais do Centro Universitário Unieuro

ISSN: $1809-1261$

UNIEURO, Brasília, número 16, 2015, pp. 46-67.

de diabetes tipo 2 em homens fumantes, de nacionalidade finlandesa e que possuíssem idade entre 50 e 69 anos.

No início do estudo, os homens foram excluídos se tabagistas de pelos menos cinco cigarros por dia; se teve uma história prévia de câncer; angina grave; insuficiência renal crônica; cirrose hepática; alcoolismo ou outras condições médicas que limitavam a participação a longo prazo. Além disso, também foram excluídos os homens que receberam terapia anticoagulante, ou que usavam vitamina $E$, vitamina $A$, ou suplemento de beta-caroteno em excesso. (MÄNNISTÖ et al, 2010, p. 2, tradução nossa)

No início da pesquisa os participantes responderam um questionário sobre histórico médico, características gerais, atividades físicas e tabagismo. Foram realizados exames de sangue, cálculo do IMC e aferição da pressão arterial. No questionário havia um item que perguntava sobre a quantidade de vezes por semana que se ingeria carne e linguiça.

Os resultados apontaram que, em média, os homens mais jovens faziam um elevado consumo de carne total e eram mais predispostos a serem mais obesos, fisicamente menos ativos e tinham mais calorias na dieta em comparação com os outros (MÄNNISTÖ et al, 2010), isso pode estar relacionado com a praticidade que as pessoas buscam na hora de se alimentarem, optando por fasts foods na maioria das vezes. Borges (2003), realizou uma pesquisa com jovens universitários para compreender seus hábitos alimentares, e como resultado observou que "o processo de escolha dos alimentos é influenciado por diversos fatores, entre eles a questão da disponibilidade de tempo, recursos financeiros, lugar, variedade de alimentos e qualidade dos produtos oferecidos".

Por meio do estudo de Männistö et al (2010), é possível constatar que o risco para diabetes tipo 2 está mais ligada ao consumo de carne processada que o de carne vermelha.

Os resultados dos estudos demonstraram que uma dieta pobre em consumo de carne processada reduz o risco de desenvolvimento de diabetes tipo 2 (MÄNNISTÖ et al, 2010; DAN et al, 2002). Uma dieta adequada é fundamental para o controle dos níveis de glicemia no sangue, como por exemplo, diminuir o açúcar dos alimentos e evitá-los quando possuem um alto nível de gordura saturada, para que a massa corporal não se eleve, pois como 
Artigo original

Hegemonia - Revista Eletrônica de Relações Internacionais do Centro Universitário Unieuro

ISSN: $1809-1261$

UNIEURO, Brasília, número 16, 2015, pp. 46-67.

relatado no estudo de Männistö et al (2010), os homens que consumiam mais calorias durante as refeições eram os mais obesos e mais inativos fisicamente, sendo a obesidade um dos fatores de risco para o desenvolvimento da diabetes tipo 2.

A obesidade é um fator de risco para a diabetes tipo 2, pois ela é caracterizada pelo excesso de gordura corporal e também é definida por ter um IMC igual ou maior a $30 \mathrm{~kg} / \mathrm{m}^{2}$, que significa, ter sobrepeso grau II (ESCOBAR, 2009; TAVARES; ANJOS, 1999). Sartorelli e Franco (2003, p. 32), confirmam o que Escobar (2009), diz sobre a obesidade e ainda faz a correlação com a diabetes tipo 2 quando menciona que "estima-se que entre 80 e $90 \%$ dos indivíduos acometidos por esta doença são obesos e o risco está diretamente associado ao aumento do índice de massa corporal". Francischi et al (2000), dizem que "no desenvolvimento de diabetes, o tecido adiposo atua aumentando a demanda por insulina e, em pacientes obesos, criando resistência à esta, 0 que ocasiona aumento na glicemia e consequente hiperinsulinemia".

De acordo com Dullius (2007), o diabético deve estar em constante vigilância à manutenção do peso, para que a estrutura corporal esteja saudável para as condições de saúde. Ou seja, o indivíduo tem de estar atento ao que come para que sua dieta não prejudique a situação da diabetes, a prática de atividades físicas também é de fundamental importância para que o peso corporal não se eleve. Ressalta-se que, também há opções que fazem com que o risco de desenvolvimento de diabetes tipo 2 seja atenuado, a exemplo de evitar carne processada, como relatam os estudos de Dan et al (2002) e Männistö et al, (2010).

Dullius (2007), diz que uma alimentação saudável abrange a diminuição do consumo de gorduras de origem animal, alimentos industrializados, produtos ricos em sal e carboidratos. Recomenda ainda que seja elevado o consumo de alimentos naturais.

Um estudo realizado por Biase et al (2005), que teve como base a comparação dos valores de triglicerídeos, colesterol total, LDL e HDL entre pessoas vegetarianas e onívoras, apontou como resultado, que os onívoros tinham níveis de colesterol total, LDL e triglicerídios maiores que os dos vegetarianos. $\mathrm{E}$ a partir deste estudo é possível correlacionar que uma dieta vegetariana seja recomendável tanto para a prevenção da diabetes tipo 2 quanto para mantê-la controlada.

A American Diabetes Association - ADA (2013), traz que muitas pessoas 
Artigo original

Hegemonia - Revista Eletrônica de Relações Internacionais do Centro Universitário Unieuro

ISSN: $1809-1261$

UNIEURO, Brasília, número 16, 2015, pp. 46-67.

estão optando por seguir dietas vegetarianas atualmente. E a Sociedade Vegetariana Brasileira - SVB (2014) define como vegetarianismo o regime alimentar que exclui todos os tipos de carne.

A ADA (2013), declara que a dieta vegetariana é uma opção saudável para todas as pessoas, inclusive para os que têm diabetes, pois esse tipo de regime alimentar colabora na prevenção e controle da doença. Isto devido ao alto teor de fibras ingeridas e a inclusão de vários tipos de alimentos de origem vegetal como legumes, frutas, verduras, feijões e cereais nas refeições. Uma dieta vegetariana faz com que o indivíduo consuma um teor de gordura saturada baixa, sendo livre de colesterol quando comparada a dieta onívora. (ADA, 2013).

Em suma, neste tópico discute-se e constata-se que os artigos encontrados por meio da busca bibliográfica apontam que o risco para desenvolvimento de diabetes tipo 2 está diretamente relacionado ao consumo frequente de carne processada. Nesse sentido, recomenda-se mais ações informativas acerca dos malefícios deste tipo de alimento e sua associação com o desenvolvimento de diabetes tipo 2 .

\section{Câncer}

Os artigos selecionados para este estudo apontam vários tipos de cânceres relacionados ao consumo frequente e excessivo de carne vermelha e processada. A maioria dos estudos era de coorte prospectivo ou de casocontrole, e segundo Willett (1997), os estudos de coorte são mais utilizados nessas pesquisas, pois acompanham os participantes durante anos, sendo possível detectar as incidências de doenças relacionadas a um fator de risco.

Dentre os cânceres, o de colorretal foi o mais encontrado nas referências (CROSS et al, 2007; SILVEIRA et al, 2008; SOUZA, et al 2013; GUIMARÃES et al 2013), e em comum, foi relatado que as pessoas diagnosticadas com este câncer foram as que ingeriram maior quantidade de carne vermelha $e$ processada e que não consumiam muitos vegetais, fibras, legumes e frutas, quando comparado com as que tinham uma alimentação mais saudável.

De acordo com o INCA (2014), "o câncer colorretal abrange tumores que acometem um segmento do intestino grosso (o cólon) e o reto. É tratável e, na maioria dos casos, curável, ao ser detectado precocemente, quando ainda não 
Artigo original

Hegemonia - Revista Eletrônica de Relações Internacionais do Centro Universitário Unieuro

ISSN: $1809-1261$

UNIEURO, Brasília, número 16, 2015, pp. 46-67.

se espalhou para outros órgãos". Para Guimarães et al (2013), o alto índice de mortalidade deste câncer está ligado ao diagnóstico tardio da doença.

Segundo Souza, et al (2013), o câncer colorretal é o terceiro mais comum na população masculina e o segundo na população feminina. O INCA (2014), identificou que no Brasil 32.600 pessoas foram notificados no ano de 2014, sendo 15.070 homens e 17.530 mulheres. As pessoas que moram nas periferias, que possuem um baixo nível de escolaridade, inativas fisicamente, que consomem um alto índice de carne vermelha frita e carne processada são as mais propensos a desenvolverem este tipo de câncer (SOUZA, et al 2013).

O resultado supracitado, pode ser explicado por Buss e Filho (2007, p. 78) quando dizem que "os determinantes sociais em saúde são os fatores sociais, econômicos, culturais, étnicos/raciais, psicológicos e comportamentais que influenciam a ocorrência de problemas de saúde". Sendo assim, a alimentação e moradia configuram-se como sendo determinantes de saúde, pois a condição de onde se vive e o que se come implicam nos fatores de risco associados a doenças.

Para Guimarães et al (2013), a renda não interfere de forma direta sobre o câncer colorretal, porém pode colaborar, visto que promove determinantes sociais, contribui na exposição de risco (dieta adequada ou inadequada) e atua no diagnóstico precoce ou tardio da doença.

Em um estudo de coorte prospectivo, constatou-se que um estilo de vida inadequado, que consiste em inatividade física, consumo de álcool, tabagismo, consumo frequente de carne vermelha e processada e o baixo consumo de vegetais e frutas contribui para o desenvolvimento de câncer colorretal (SOUZA, et al 2013).

Cross et al, (2007), mencionam que em a ingestão de carne vermelha gera mais predisposição para o desenvolvimento de câncer do reto.

Hughes et al (2000), seguindo uma mesma linha em seu estudo buscar explicar a associação entre o câncer colorretal e a carne vermelha. Segundo o autor, o aumento do metabolismo de proteínas no cólon, que devido aos produtos finais desse processo metabólico provocam a formação de fenóis, sulfitos, indóis, amônia, nitratos e aminas, estes compostos sugerem um efeito tóxico sobre a mucosa intestinal gerando a predisposição para o surgimento das células cancerígenas (HUGHES et al, 2000).

De acordo com o INCA (2014), a prevenção do câncer colorretal está baseada numa dieta que seja rica em vegetais, e que sejam pobres em gordura 
Artigo original

Hegemonia - Revista Eletrônica de Relações Internacionais do Centro Universitário Unieuro

ISSN: $1809-1261$

UNIEURO, Brasília, número 16, 2015, pp. 46-67.

saturada, com práticas de atividades físicas regulares e a redução do consumo exagerado de carne vermelha, tendo em vista que se o individuo tem mais de 50 anos, e possui um histórico familiar do câncer é significativamente maior a chance de ter a doença quando o mesmo é sedentário e/ou obeso. Assim também relata Willett (1997), que uma alimentação natural com maior consumo de vegetais pode reduzir consideravelmente o risco de muitos cânceres.

O câncer de esôfago, foi o segundo mais encontrado nas referências (CROSS et al, 2007; SILVEIRA et al 2008; STEFFEN et al, 2012).

Estudo de Silveira et al (2008), dizem que os sujeitos que possuem câncer no esôfago, são os que tendem a relatar uma maior ingestão de carnes vermelhas e laticínios ricos em gordura e um baixo consumo de frutas, verduras e grãos integrais. No estudo, os alimentos que não foram associados ao câncer de esôfago foram: frutas não cítricas e vegetais crus. Sendo que, as carnes vermelhas e produtos lácteos ricos em gordura foram positivamente associados à doença.

Foi possível identificar que os indivíduos que consumiam grandes quantidades de carne, eram os mais propensos a ter um IMC elevado, ser fumantes, e consumiam pequenas quantidades de frutas. (STEFFEN et al, 2012).

O INCA (2014), aponta que no Brasil, no ano de 2014 foram relatados 10.780 casos ao total, sendo 8.010 homens e 2.770 mulheres.

O tipo de câncer no esôfago mais frequente é o carcinoma epidermoide escamoso, responsável por $96 \%$ dos casos. Silveira et al (2008), apontam em seu estudo que as pessoas diagnosticadas com esse câncer relataram consumir menos frutas e vegetais e mais carne vermelha.

A prevenção para o câncer de esôfago está ligada a uma dieta que seja rica em frutas e legumes, e com a redução do consumo frequente de bebidas muito quentes, alimentos defumados, bebidas alcoólicas e derivados do tabaco (INCA, 2014).

O estudo de Cross et al (2007), menciona que não houve associação entre a ingestão de carne vermelha e câncer de mama, ao contrário do estudo de Lima et al (2008), que faz referência que a carne vermelha está positivamente associada ao risco de câncer de mama, devido sua composição nutricional que é rica em gordura saturada. Já as carnes processadas são 
Artigo original

Hegemonia - Revista Eletrônica de Relações Internacionais do Centro Universitário Unieuro

ISSN: $1809-1261$

UNIEURO, Brasília, número 16, 2015, pp. 46-67.

associadas porque contêm potenciais agentes cancerígenos, sendo um deles a nitrosamina ${ }^{6}$ (LIMA et al 2008).

Por meio do estudo de caso-controle de Lima et al (2008), realizado no Nordeste do Brasil, foi possível correlacionar a ingestão de alimentos lácteos, ovos, feijão e frutas a um risco reduzido do desenvolvimento de câncer de mama. $O$ consumo de carne vermelha foi associada ao câncer, enquanto 0 consumo de verduras foi ligado a um possível fator protetor contra o risco de desenvolvimento da doença.

Uma das hipóteses que sustenta a ideia de que o consumo de verduras pode ser um possível protetor contra o câncer, é que em um estudo realizado por Pool-Zobel et al (1997), foi considerado que legumes, frutas e verduras a exemplo do espinafre, tomate e cenoura, podem proteger contra o risco de câncer, tendo em vista que esses alimentos possuem os carotenoides, que exercem efeito protetor contra o câncer por meio da redução de lesões ao DNA.

Lima et al (2008), enfatizam que como o estudo foi realizado no Nordeste do Brasil, não é recomendável que os resultados sejam generalizados nem expandidos para outras localidades, tendo em vista que cada região possui um hábito alimentar diferente, e que o câncer de mama foi relacionado à população desta região específica.

Segundo o INCA, no ano de 2014, foram notificados no Brasil 57.120 casos de câncer de mama, no entanto este diagnóstico ainda é tardio como mencionado a seguir:

Segundo tipo mais frequente no mundo, o câncer de mama é o mais comum entre as mulheres, respondendo por $22 \%$ dos casos novos a cada ano. Se diagnosticado e tratado oportunamente, o prognóstico é relativamente bom. No Brasil, as taxas de mortalidade por câncer de mama continuam elevadas, muito provavelmente porque a doença ainda é diagnosticada em estágios avançados. (INCA, 2014).

Nitrosaminas são produzidas a partir da reação entre nitritos e aminas. São compostos orgânicos que possuem agentes mutagênicos, teratogênicos e principalmente carcinogênicos, caracterizam-se por serem altamente perigosos para a saúde humana. Elas são adicionadas a carne para que, esta se mantenha conservada e com a cor avermelhada. (ANTÓN; LIZASO, 2001) 
Artigo original

Hegemonia - Revista Eletrônica de Relações Internacionais do Centro Universitário Unieuro

ISSN: $1809-1261$

UNIEURO, Brasília, número 16, 2015, pp. 46-67.

Evitar a obesidade, manter dieta equilibrada e saudável, praticar exercícios regularmente e diminuir a ingestão de álcool são algumas das prevenções para o câncer de mama (INCA, 2014).

Outros cânceres como: de pulmão, laringe e faringe (CROSS et al, 2007; STEFFEN et al, (2012), também foram associados a um consumo de carne vermelha e processada. $O$ risco é significativamente maior quando o indivíduo é fumante ou ex-fumante.

Cross et al (2007), avaliam que no estudo deles, as mulheres que tinham um consumo maior de carne processada tinham um elevado risco de serem diagnosticadas com câncer do colo do útero. E que para os homens esse mesmo consumo elevado era relativamente ligado ao câncer de próstata.

Este tópico apresenta os cânceres que através de evidências científicas, mostram ter correlação com o consumo de carne vermelha e processada. A etiologia dos cânceres normalmente está associada a fatores hereditários e a fatores externos, como por exemplo: consumo de cigarro pode causar câncer no pulmão, e exposição ao sol pode ocasionar câncer de pele. Entretanto, estudos têm demonstrado que a alimentação está diretamente associada tanto ao possível desenvolvimento de câncer, como para a sua prevenção.

Doenças Cardiovasculares

Segundo Rique et al (2002), as doenças cardiovasculares são consideradas a principal causa de mortalidade no mundo, e que nos países em desenvolvimento essa taxa tende a ser maior. Para esses autores as doenças cardiovasculares "são influenciadas por um conjunto de fatores de risco, alguns modificáveis mediante alterações no estilo de vida, como a dieta adequada e o exercício regular" (RIQUE et al, 2002, p. 244).

Nas pesquisas realizadas por Sinha et al (2009), e Ashaye et al (2011), foram encontrados como resultados em um estudo de coorte, que há uma prevalência maior de doenças cardiovasculares nas pessoas que relataram ingerir frequentemente carne vermelha e processada e faziam um consumo pobre em verduras, frutas e legumes. 
Artigo original

Hegemonia - Revista Eletrônica de Relações Internacionais do Centro Universitário Unieuro

ISSN: $1809-1261$

UNIEURO, Brasília, número 16, 2015, pp. 46-67.

As pessoas que eram fumantes, por inalarem produtos químicos cancinogênicos, e que consumiam compostos nitrosos através das carnes processadas tiveram 0 maior índice de mortalidade por doenças cardiovasculares (SINHA et al, 2009). Ainda neste estudo, as pessoas que faziam pouca ingestão de carne vermelha tiveram um risco diminuído de mortalidade por doenças cardiovasculares.

Ashaye et al (2011), constatou que o consumo frequente de carne vermelha estava associado a um maior IMC, e eram mais frequente nos fumantes. $\mathrm{O}$ consumo de carne vermelha foi associado ao risco aumentado de insuficiência cardíaca e houve também uma associação com este fator de risco e infarto do miocárdio.

Os estudos mostraram ainda que se houvessem um consumo reduzido de carne vermelha e processada o risco de mortalidade por doenças cardiovasculares seria significativamente reduzido (SINHA et al, 2009; ASHAYE et al, 2011).

A insuficiência cardíaca encontrada nos estudo de Ashaye et al (2011), tem uma prevalência maior nas pessoas que relataram ter um consumo médio de 4 a 5 porções de carne vermelha durante a semana, e as pessoas que possuíam idade acima de 60 anos eram mais propensas a ter a doença.

Hipertensão, aterosclerose, aneurisma da aorta, outras doenças das artérias, arteríolas e capilares, foram as doenças encontradas nos integrantes do estudo de coorte que consumiam frequentemente carne vermelha $\mathrm{e}$ processada (SINHA et al, 2009).

A American Heart Association (AHA) no ano de 2014, diz que a insuficiência cardíaca pode ser tratada com estratégias para melhorar os sintomas, sendo uma delas a mudança de hábitos alimentares e atividades físicas, e diz também que as pessoas portadoras de insuficiência cardíaca, têm que ser ver como parte ativa para a melhoria das suas condições de saúde, assim como fazer consultas e exames com médicos especializados e de forma periódica.

Teixeira et al (2007), analisou por meio de um estudo de coorte, o risco cardiovascular entre vegetarianos e onívoros, e assim como os estudos selecionados, este também teve como resultado que uma alimentação 
Artigo original

Hegemonia - Revista Eletrônica de Relações Internacionais do Centro Universitário Unieuro

ISSN: $1809-1261$

UNIEURO, Brasília, número 16, 2015, pp. 46-67.

desbalanceada, com excesso de gordura de origem animal, está diretamente relacionado à doenças, principalmente as cardiovasculares. E que uma alimentação rica em produtos de origem vegetal e pobre em origem animal pode funcionar como prevenção e controle para os fatores de risco de problemas cardiovasculares.

As doenças cardiovasculares são as que comumente, as pessoas associam ao consumo de carne vermelha, ao contrário da diabetes tipo 2 e de cânceres. Sendo assim, faz-se necessário uma maior divulgação e orientação com relação ao tema para o desenvolvimento de um consumo consciente e reduzido de carne vermelha e processada, pois como comprovado pelos estudos há uma associação direta deste fator de risco com o desenvolvimento dessas DCNT.

Percebe-se que as orientações e informações de prevenção e controle destas DCNT são mais voltadas para a prática de atividades físicas, redução de açúcares e carboidratos, sendo negligenciado que o consumo de carne tem um papel significante no desencadeamento destas doenças.

\section{CONSIDERAÇÕES FINAIS}

A associação entre o consumo de carne vermelha e processada e o desenvolvimento de diabetes tipo 2, doenças cardiovasculares e cânceres, pode ser constatada por meio dos artigos científicos selecionados e aqui explorados.

Este estudo visa oferecer mais informações para que as pessoas possam compreender de forma geral que a carne vermelha e processada influencia no desencadeamento de DCNT, e que além de práticas de exercícios regulares, consumo moderado de álcool e cigarro, faz-se necessário que consumam a carne de forma mais consciente a fim de que não sejam acometidas pelas DCNT. Dessa forma, espera-se que esse estudo possa contribuir com informações acerca da problemática.

O levantamento dos artigos nas bases de dados demonstrou que o assunto não é tão abordado na literatura brasileira, sendo mais amplamente discutido no idioma inglês.

Observa-se que muitos brasileiros consomem carne vermelha $\mathrm{e}$ processada diariamente, e a questão é que as informações sobre os malefícios 
Artigo original

Hegemonia - Revista Eletrônica de Relações Internacionais do Centro Universitário Unieuro

ISSN: $1809-1261$

UNIEURO, Brasília, número 16, 2015, pp. 46-67.

da carne não chegam a todos, devido também, a indústria que está por detrás de todo o comércio destes alimentos.

Em suma, este, traz evidências científicas que o consumo de carne vermelha e processada pode ser um fator de risco para DCNT, fomenta-se então, que as pessoas passem a buscar estilos de vida mais saudáveis, fazendo uma ingestão reduzida das carnes mencionadas e que sobreponha isto com outros hábitos saudáveis, como a prática de atividade física.

A formação em Saúde Coletiva capacita o profissional sanitarista, para a formulação de políticas públicas, programas e ações no setor saúde, agindo nos fatores de risco que ameacem a saúde da população, e assim atuando na prevenção de doenças e promoção da saúde.

\section{REFERÊNCIAS}

AMERICAN DIABETES ASSOCIATION. Meal Planning for Vegetarians. 2013. Disponível em: <http://www.diabetes.org/food-and-fitness/food/planningmeals/meal-planning-for-vegetarians/?loc=ff-slabnav>. Acesso em: 21 maio 2015.

AMERICAN HEART ASSOCIATION. Prevention Treatment of Heart Failure. Disponível em: <http://www.heart.org/HEARTORG/Conditions/HeartFailure/PreventionTreatm ntofHeartFailure/Prevention-TreatmentofHeartFailure_UCM_002048_Article.jsp.> Acesso em: 20 maio 2015.

ANTÓN, Almudena; LIZASO, JESÚS. NITRITOS, NITRATOS Y NITROSAMINAS. Madrid, p.1-7, 2001. Disponível em: <http://mie.esab.upc.es/ms/formacio/Control\%20\%20Contaminacio\%20Agric ultura/biblio/nitratos\%20y\%20nitrosaminas.pdf. Acesso em: 25 maio 2015.

ASHAYE, Ajibade et al. Red meat consumption and risk of heart failure in male physicians. Nutr Metab Cardiovasc Dis, p. 941-946, dec. 2011.

ASSUNÇÃO, Maria Cecília Formoso et al. Consumo de carnes por adolescentes do Sul do Brasil. Revista de Nutrição, Campinas, v. 25, n. 4, jul./ago. 2012. 
Artigo original

Hegemonia - Revista Eletrônica de Relações Internacionais do Centro Universitário Unieuro

ISSN: $1809-1261$

UNIEURO, Brasília, número 16, 2015, pp. 46-67.

BIASE, Simone Grigolleto de et al. Dieta vegetariana e níveis de colesterol e triglicerídeos. São Paulo, p. 35-39 2005.

BORGES, Claudia Moreira. Hábitos Alimentares dos estudantes universitários: um estudo qualitativo. Campo Grande, p. 1-11, 2003.

BRASIL. Ministério da Saúde. Instituto Nacional De Câncer José de Alencar Gomes da Silva (INCA). Câncer: A informação pode salvar vidas, 2012.

. Ministério da Saúde. Instituto Nacional De Câncer José de Alencar Gomes da Silva (INCA). Câncer Colorretal. Disponível em: <http://www2.inca.gov.br/wps/wcm/connect/tiposdecancer/site/home/colorre tal/definicao+>. Acesso em: 20 maio 2015.

- Ministério da Saúde. Instituto Nacional De Câncer José de Alencar Gomes da Silva (INCA). Políticas e Ações para Prevenção do Câncer no Brasil: Alimentação, Nutrição e Atividade Física, 2009.

- Ministério da Saúde. Instituto Nacional De Câncer José de Alencar Gomes da Silva (INCA). Prevenção do Câncer Colorretal. Disponível em: $<$ http://www2.inca.gov.br/wps/wcm/connect/tiposdecancer/site/home/colorre tal/prevencao>. Acesso em: 2 maio 2015.

- Ministério da Saúde. Instituto Nacional de Câncer José de Alencar Gomes da Silva (INCA). Prevenção do Câncer de Esôfago. Disponível em: $<$ http://www2.inca.gov.br/wps/wcm/connect/tiposdecancer/site/home/esofag o/prevencao >. Acesso em: 2 maio 2015.

.Ministério da Saúde. Secretaria de Atenção à Saúde. Coordenação Geral da Política de Alimentação e Nutrição. Guia alimentar para a população brasileira: promovendo a alimentação saudável, 2006b.

. Ministério da Saúde. Secretaria de Atenção à Saúde. Departamento de Atenção Básica. Política Nacional de Alimentação e Nutrição. 2012

BUSS, Paulo Marchiori; FILHO, Alberto Pellegrini. A Saúde e seus determinantes sociais. Rev. Saúde Coletiva, Rio de Janeiro, p. 77-3, 2007.

CROSS, Amanda et al. A Prospective Study of Red and Processed Meat Intake in Relation to Cancer Risk. PLoS Medicine, v. 4, p. 1973-1984, dec. 2007.

DAM, Rob Van et al. Dietary fat and meat intake in relation to risk of type 2 diabetes in men. Diabetes Care, v. 25, n. 3, p. 417-424, mar 2002.

DULLIUS, Jane. Diabetes Mellitus: Saúde, educação, atividades físicas. Brasília, Editora Universidade de Brasília: Finatec, p. 4202007. 


\section{Artigo original}

Hegemonia - Revista Eletrônica de Relações Internacionais do Centro Universitário Unieuro

ISSN: $1809-1261$

UNIEURO, Brasília, número 16, 2015, pp. 46-67.

DUNCAN, Bruce Bartholow et al. Doenças Crônicas Não Transmissível: prioridade para enfrentamento e investigação. Rev. Saúde Pública. Porto Alegre, RS. p. 126-134, 2012.

ESCOBAR, Fernanda de Almeida. Relação entre Obesidade e Diabete Mellitus Tipo II em Adultos. Cadernos UniFoa, p. 69-72, 2009. Disponível em: <http://web.unifoa.edu.br/cadernos/edicao/11/69.pdf>. Acesso em: $12 \mathrm{abr}$ 2015.

FRANCISCHI, Rachel Pamfilio Prado de et al. Obesidade: atualização sobre sua etiologia, morbidade e tratamento. Rev. Nutr, v. 13, n. 1, p. 17-28, 2000.

GIL, Antônio. Métodos e técnicas de pesquisa social. 6a edição. São Paulo: Atlas, 2008

GOMES, Fábio da Silva. Carotenóides: uma possível proteção contra o desenvolvimento de câncer. Revista de Nutrição, Campinas, p. 537-548, set./out.2007.

GUIMARÃES, Raphael Mendonça et al. Increase income and mortality of colorrectal cancer in brazil, 2001-2009. Arq. Gastroenterol, São Paulo, v. 50, n. 1, jan./mar. 2013.

HUGHES, Roisin et al. Protein Degradation in the Large Intestine: Relevance to Colorectal Cancer. Horizon Scientific Press, p. 51-58, 2000. Disponível em: <http://www.horizonpress.com/backlist/ciim/v/v1/05.pdf>. Acesso em: 25 maio 2015.

KUSUMOTA, Luciana; RODRIGUES, Rosalina Aparecida Partezani; MARQUES, Sueli. Idosos com insuficiência renal crônica: alterações do estado de saúde. Rev. Latino-am Enfermagem. São Paulo, p. 525-532, maio/jun. 2004.

LIMA, Flávia Emília Leite de et al. Diet and cancer in Northeast Brazil: evaluation of eating habits and food group consumption in relation to breast câncer. Cad. Saúde Pública, Rio de Janeiro, p. 820-828, apr. 2008.

MÄNNISTÖ, Satu et al. High processed meat consumption is a risk factor of type 2 diabetes in the ATBC study. Br J Nutr, p. 1817-1822, jun. 2010.

MEDEIROS, Carla Campos Muniz et al. Prevalência dos fatores de risco para diabetes mellitus de servidores públicos. Revista Eletrônica de Enfermagem, p. 1-11, jul./set. 2012. Disponível em: 
Artigo original

Hegemonia - Revista Eletrônica de Relações Internacionais do Centro Universitário Unieuro

ISSN: $1809-1261$

UNIEURO, Brasília, número 16, 2015, pp. 46-67.

<https://www.fen.ufg.br/fen_revista/v14/n3/pdf/v14n3a12.pdf>. Acesso em: 13 maio 2015

MELLO, Elsa et al. Obesidade infantil: como podemos ser eficazes? Jornal de Pediatria, v. 80, n. 3, p. 173-182, 2004.

MOTA, Elizabeth Duarte. Alimentação Natural: uma opção que faz diferença. Petrópolis: Vorazes, 2005.

ORTIZ, Ana Carolina Alves; ZANETTI, Maria Lúcia. Levantamento dos fatores de risco para diabetes mellitus tipo 2 em uma insituição de ensino superior. Rev. Latino-Enfermagem. São Paulo, p. 58-63. 2001.

PAN, An et al. Red meat consuption and mortality. American Medical Association, p. 555-563, abr. 2012.

PITANGA, Francisco José Gondim. Epidemiologia, atividade física e saúde. Rev. Bras. Ciên. e Mov. Brasília. v. 10, n 03, p. 49-54, jul. 2002.

POLL-ZOBEL, B. L. Consumption of vegetables reduces genetic damage in humans: first results of a human intervention trial with carotenoid-rich foods. Oxford University, v.18, n.9, p.1847-1850, 1997. Disponível em: <http://carcin.oxfordjournals.org/content/18/9/1847.long>. Acesso em: 5 jun. 2015.

RIQUE, Ana Beatriz Ribeiro et al, Nutrição e exercício na prevenção e controle das doenças cardiovasculares. Rev Bras Med Esporte, v. 8, n. 6, p. 1-11, Nov./dez. 2002. Disponível em: <http://www.scielo.br/pdf/\%0D/rbme/v8n6/v8n6a06.pdf>. Acesso em: 23 maio 2015.

SARTORELLI, Daniela Saes; FRANCO, Laércio Joel. Tendências do diabetes mellitus no Brasil: o papel da transição nutricional. Caderno de Saúde Pública, Rio de Janeiro, p. s29-s36, 2003.

SCHRAMM, Joyce Mendes de Andrade. Transição epidemiológica e o estudo de carga de doença no Brasil. Rio de Janeiro, p. 897- 908. 2004.

SILVEIRA, Navarro et al. Food group intake and risk of subtypes of esophageal and gastric câncer. Int J Cancer, p. 825-560, aug. 2008.

SINHA, Rashmi et al. Meat intake and mortality: a prospective study of over half a million people. Arch Intern Med, p. 562-571, mar. 2009. 
Artigo original

Hegemonia - Revista Eletrônica de Relações Internacionais do Centro Universitário Unieuro

ISSN: $1809-1261$

UNIEURO, Brasília, número 16, 2015, pp. 46-67.

SOCIEDADE VEGETARIANA BRASILEIRA. Disponível em: $<$ http://www.svb.org.br/vegetarianismo1/o-que-e>. Acesso em: 23 maio 2015.

SOUZA, Vanina Cordeiro de et al. Relationship between nutritional status and immediate complications in patients undergoing colorectal surgery. Journal of Coloproctology, p. 83-91, may. 2013.

STEFFEN, Annika et al. Meat and heme iron intake and risk of squamous cell carcinoma of the upper aero-digestive tract in the European Prospective Investigation into Cancer and Nutrition (EPIC). Cancer Epidemiol Biomarkers Prev, p. 2138-2148, dec. 2012.

TEIXEIRA, Rita de Cássia Moreira de Almeida et al. Estado nutricional e estilo de vida em vegetarianos e onívoros - Grande Vitória - ES. Revista Bras. Epidemiologia, p. 131-143, 2007.

WILLET, Walter. Nutrition and câncer. Salud pública, México, v. 39, n. 4, jul. 1997. 